\title{
Performance of optical and hybrid joint transform correlators for tasks of optical security
}

\author{
L.I. Muravsky ${ }^{1)}$, Ya.P. Kulynych ${ }^{1)}$, O.P. Maksymenko(), T.I. Voronyak ${ }^{1)}$, \\ I.Y. Pogan'1), F.L. Vladimirov'2), S.A. Kostyukevych ${ }^{3)}$, V.M. Fitio() \\ ${ }^{1}{ }^{1}$ Karpenko Physico-Mechanical Institute, NAS of Ukraine, 5 Naukova Str., 79601 Lviv, Ukraine; \\ Phone: +380 (322) 63 3388; e-mail: dep24@ah.ipm.lviv.ua \\ ${ }^{2}$ S.I.Vavilov State Optical Institute, 12 Birjevaya liniya, 199034 St. Petersburg, Russia; \\ Phone: +88(12)1510852; e-mail:vlv@fl.spb.ru \\ ${ }^{3}$ Institute of Semiconductor Physics, NAS of Ukraine, 45 prospect Nauky, 03028 Kiev, Ukraine; \\ Phone: +390(44)2656205; e-mail:sergeyk@isp.kiev.ua \\ ${ }^{4)}$ National University "Lvivska Polytechnika", 12 Bandera Str., 79013 Lviv, Ukraine; \\ Phone: +380(322) 727747
}

\begin{abstract}
Performance of optical and hybrid joint transform correlators (JTCs) for security verification of optical marks containing transformed phase masks (PMs) is studied. The peakto-noise ratio (PNR) and relative intensity of correlation peaks are selected as basic criteria for comparative analysis of such two types of JTCs. The theoretical model of an interference noise produced as a result of an input and reference binary PMs cross-correlation on a conventional JTC output is developed. The optically addressed spatial light modulator (OASLM) based on the chalcogenide glass photoconductor - nematic liquid crystal structure is considered. The experimental setup of an OASLM JTC is designed on the basis of this SLM. The relations a PNR and relative intensity versus an effective focal length of an optical Fourier processor are studied and the basic reasons limiting efficiency of the setup are analyzed. The optoelectronic device for identification of credit cards based on a hybrid optical-digital JTC architecture is built. The conditions of a joint power spectrum recording by a CCD-camera in this device are investigated. The concluding about expediency of OASLM JTC and optoelectronic device usage for different security applications is made.
\end{abstract}

Keywords: optical security, transformed phase mask, joint transform correlator, peak-tonoise ratio, spatial light modulator, optoelectronic device, interference noise.

Paper received 05.06.01; revised manuscript received 03.06.02; accepted for publication 25.06.02.

\section{Introduction}

Usage of optical security systems and devices based on a joint transform correlator (JTC) architecture for identification of credit cards, documents and other products is very promising. The experimental demonstrations of the random phase encoding technique for security verification show that both optical and hybrid JTCs can be successfully applied [1-4]. The comparative analysis of these JTCs types is useful for improvement the well-known methods and development the new methods for optical security. Besides such comparative analysis is useful for designing and creation of new high-performance security systems constructed on the basis of the JTC architectures. For this purpose, we have engineered the experimental setup of a JTC with an optically addressed SLM in a frequency domain (OASLM JTC) and the optoelectronic device for identification of credit cards based on a hybrid optical-digital JTC (HOD JTC) archi- tecture. In these correlators we used a random phase mask (PM) $[1,2]$ as a reference image and a transformed PM [3-6] as an input image or as an optical mark. To compare the performance of these correlators, we used the same optical marks for both JTC. Thus, a peak-to-noise ratio (PNR) as the main yardstick, with which the correlators were compared, was selected. At first, we carried out the theoretical estimation of an interference noise surrounding the correlation peak and found the PNR on the JTC output, if two binary random PMs are entered into its input. Further we studied the OASLM JTC and analysed the characteristics of the OASLM. To study the performance of the OASLM JTC, we found the PNRs and relative intensities of correlation peaks. We also examined the performance of the optoelectronic device based on a HOD JTC architecture. For this purpose, we have found the PNRs and relative intensities of peaks for different conditions of a joint power spectra recording by a CCD-camera. The performed researches have shown the 


\section{L.I. Muravsky et al.: Performance of optical and hybrid joint transform...}

two reviewed types of correlators can be used for construction of security devices.

\section{Random phase masks}

The correlation methods of optical security are based on usage of optical marks containing PMs. To study the performance of phase and transformed phase masks in a JTC, we have used analytical equations that describe beforehand known spacing of pixels in a mask. ${ }^{3-5}$ However these equations are unacceptable for random binary PMs. In this connection, we have considered a binary PM as set of spatially ordered pixels of the identical shape, the phase of which is equal to 0 or $\pi$. We considered two masks entered into the conventional JTC as input and reference images. To find expressions for variance of an interference noise and autocorrelation function on an output of a conventional JTC, we have used the formula [7] for mean square deviation of a cross-correlation function for two arbitrary unidimensional binary PMs with equal lengths. This formula is fair for a binomial distribution law of pixels in a mask. For pixels of length $a$ and with a step between adjacent pixels, equal $a$, this formula can be expressed as [7]

$$
\sigma\left(R_{\tilde{m}}-S_{\tilde{m}}\right)=a \sqrt{M-|\tilde{m}|}
$$

where $R_{\tilde{m}}$ is a quantity of a cross-correlation function terms in a point $\tilde{m} a$, equal $+1 ; S_{\tilde{m}}$ is a quantity of a cross-correlation function terms in a point $\tilde{m} a$, equal -1 ; $M$ is a quantity of pixels in each of masks, thus $m=1,2, \ldots, M$ is a number of a pixel; $\tilde{m}=0, \pm 1, \ldots, \pm\left(M_{0}-1\right), M_{0} \leq M$.

Using the formula (1), we can find expression for variance of a spatial interference noise, which one is produced along a coordinate axis $\vec{x}^{\prime}$ as a result of a crosscorrelation of two unidimensional masks of identical length. Because the complex amplitude of each pixel with a center in an origin of the axis $\vec{x}^{\prime}$ can be defined as

$$
t(x)=\operatorname{rect}\left(\frac{x}{a}\right) \exp [j \theta(x)]
$$

where $\theta(x)$ is equal 0 or $\pi$, the autocorrelation of a function (2) is

$$
t\left(x^{\prime}\right) * t\left(x^{\prime}\right)=a \Lambda\left(\frac{x^{\prime}}{a}\right)=\gamma\left(\frac{x^{\prime}}{a}\right),
$$

where $*$ stands for correlation, $\Lambda(\ldots)$ is the triangle function. If the step between adjacent pixels is equal $c$, the variance of a spatial interference noise in an arbitrary point on the interval $[(\tilde{m}-1) c,(\tilde{m}+1) c]$, arranged on the axis $\vec{x}^{\prime}$, can be expressed using Eqs (1)-(3) as

$$
\sigma^{2}[(\tilde{m}-1) c,(\tilde{m}+1) c]=(M-|\tilde{m}|) \gamma^{2}\left(\frac{x^{\prime}-\tilde{m} c}{a}\right),
$$

where $\gamma\left(\frac{x^{\prime}-\tilde{m} c}{a}\right)=a \Lambda\left(\frac{x^{\prime}-\tilde{m} c}{a}\right)$.

If the center of a spatial interference noise is coincided with the origin of the axis $\vec{x}^{\prime}$, Eq. (4) can be written as a variance of this noise in an arbitrary point of this axis, i.e.,

$$
\sigma^{2}\left(x^{\prime}\right)=\frac{1}{2 M-1} \sum_{\tilde{m}=-M+1}^{\tilde{m}=M-1}(M-|\tilde{m}|) \gamma^{2}\left(\frac{x^{\prime}-\tilde{m} c}{a}\right) .
$$

In a case, when two phase masks are identical, the interference noise is not change for all $\tilde{m}$ except for $\widetilde{m}=0$, and Eq. (5) can be written as

$$
\begin{aligned}
& \sigma^{2}\left(x^{\prime}\right)=\frac{1}{(2 M-1)-1} \times \\
& \times\left[\sum_{\tilde{m}=-M+1}^{\tilde{m}=M-1}(M-|\tilde{m}|) \gamma^{2}\left(\frac{x^{\prime}-\tilde{m} c}{a}\right)-M \gamma^{2}\left(\frac{x^{\prime}}{a}\right)\right]
\end{aligned}
$$

The autocorrelation peak is produced within the limits of the interval $[-c, c]$. The amplitude distribution of this peak can be represented as

$$
P\left(x^{\prime}\right)=M \gamma\left(\frac{x^{\prime}}{a}\right) \text {. }
$$

If two different two-dimensional binary random phase masks with equal dimensions $M \times N$ are placed apart $2 y_{0}$ one from another in an input of a conventional JTC, the variance of the interference noise in a diffraction center (DC) of a JTC output plane $\left(x^{\prime}, y^{\prime}\right)$ allowing to (5) is given by

$$
\begin{aligned}
& \sigma^{2}\left(x^{\prime}, y^{\prime}\right)=\frac{1}{(2 M-1)(2 N-1)} \times \\
& \times\left[\sum _ { \tilde { m } = - M + 1 } ^ { \tilde { m } = M - 1 } \sum _ { \tilde { n } = - N + 1 } ^ { \tilde { n } = N - 1 } ( M - | \tilde { m } | ) \left(N-|\tilde{n}| \gamma^{2}\left(\frac{x^{\prime}-\tilde{m} c}{a}, \frac{y^{\prime}-\tilde{n} d}{b}\right)-\right.\right. \\
& \left.-2 M N \gamma^{2}\left(\frac{x^{\prime}}{a}, \frac{y^{\prime}}{b}\right)\right]
\end{aligned}
$$

where $M$ and $N$ are the quantities of columns and rows in these masks; $m=1,2, \ldots, M$ is a number of a column; $n=1,2, \ldots, N$ is a number of a row; $\tilde{m}=0, \pm 1, \ldots, \pm\left(M_{0}-1\right) ; M_{0} \leq M ; \tilde{n}=0, \pm 1, \ldots, \pm\left(N_{0}-1\right)$; $N_{0} \leq N ; a$ is a size of a pixel along the axis $\vec{x} ; b$ is a size of a pixel along the axis $\vec{y} ; c$ and $d$ are a width and high of a rectangular cell $c \times d$ of a phase mask, in which the pixel is arranged (see Fig.1).

The expression for the noise variance in a \pm 1 -st diffraction order of a JTC output becomes

$$
\sigma^{2}\left( \pm x^{\prime}, \pm y^{\prime}\right)=\frac{1}{(2 M-1)(2 N-1)} \times
$$

$$
\begin{aligned}
& \times \sum_{\tilde{m}=-M+1}^{\tilde{m}=M-1} \sum_{\tilde{n}=-N+1}^{\tilde{n}=N-1}(M-|\tilde{m}|)(N-|\tilde{n}|) \times \\
& \times \gamma^{2}\left(\frac{ \pm x^{\prime}-\tilde{m} c}{a}, \frac{ \pm y^{\prime}-\tilde{n} d-2 y_{0}^{\prime}}{b}\right)
\end{aligned}
$$$$
\text { where } y_{0}^{\prime}=y_{0} \text {. }
$$ 


\section{L.I. Muravsky et al.: Performance of optical and hybrid joint transform...}

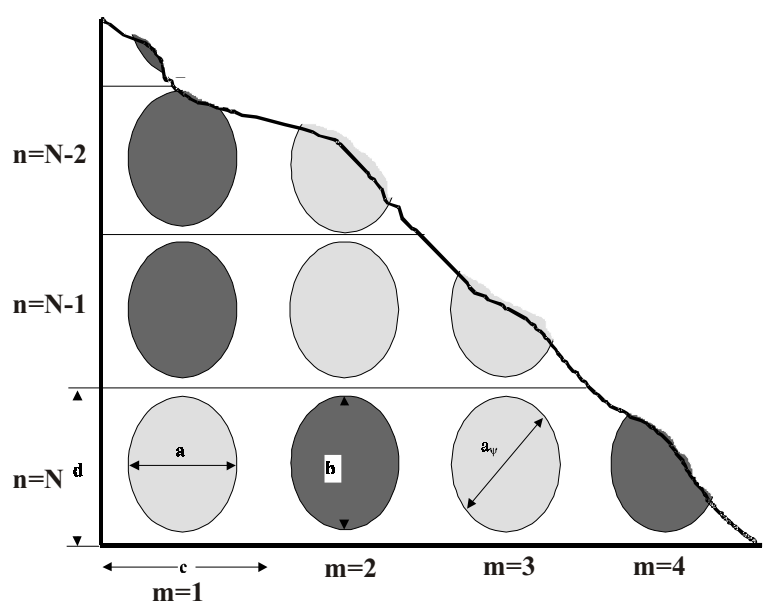

Fig. 1. Fragment of a phase mask containing a rectangular grating of pixels.

If two identical phase masks are entered to a conventional JTC, the noise variance in a the \pm 1 -st order can be defined as (see Eq. (6))

$$
\begin{aligned}
& \sigma^{2}\left( \pm x^{\prime}, \pm y^{\prime}\right)=\frac{1}{(2 M-1)(2 N-1)} \times \\
& \times \sum_{\tilde{m}=-M+1 \tilde{n}=-N+1}^{\tilde{m}=M-1} \sum_{\tilde{n}=N-1}(M-|\tilde{m}|)(N-|\tilde{n}|) \times \\
& \times \gamma^{2}\left(\frac{ \pm x^{\prime}-\tilde{m} c}{a}, \frac{ \pm y^{\prime}-\tilde{n} d-2 y_{0}^{\prime}}{b}\right),
\end{aligned}
$$

where $y_{0}^{\prime}=y_{0}$. Using Eq. (7), the correlation peak amplitude in the point $\left(0, \pm 2 y_{0}\right)$ is given by

$$
P_{ \pm}\left(0, \pm 2 y_{0}^{\prime}\right)=M N \gamma(0,0) \text {. }
$$

Let both identical reference and input PMs are displayed simultaneously in the input plane of a conventional JTC. We can estimate influencing dimension of such masks on the PNR on the output of a correlator. Using Eqs (8) and (9), the PNR can be expressed as

$$
\begin{aligned}
& \mu=\frac{P_{ \pm}^{2}\left(0, \pm 2 y_{0}^{\prime}\right)}{\sigma^{2}\left( \pm \tilde{m} c, \pm \tilde{n} d \pm 2 y_{0}^{\prime}\right)}=\frac{I\left(0, \pm 2 y_{0}^{\prime}\right)}{\sigma^{2}\left( \pm \tilde{m} c, \pm \tilde{n} d \pm 2 y_{0}^{\prime}\right)}= \\
& =(M N)^{2} \gamma^{2}(0,0) /\left\{\frac{1}{(2 \tilde{M}+1)(2 \tilde{N}+1)-1} \times\right. \\
& \left.\times\left[\sum_{\tilde{m}=-\tilde{M} 1 \tilde{n}=-\tilde{N}}^{\tilde{m}=\tilde{n}} \sum_{\tilde{N}}^{\tilde{N}}(M-|\tilde{m}|)(N-|\tilde{n}|)-M N\right]\right\},
\end{aligned}
$$

where $\tilde{m}=0,1, \ldots, \tilde{M} ; \tilde{n}=0,1, \ldots, \tilde{N}$

The dependencies PNR versus dimension of a window $(2 \tilde{M}+1) \times(2 \tilde{N}+1)$ limiting readouts of a noise are obtained from Eq. (10) and represented in Fig. 2 for different $M$ (if $\tilde{M}=\tilde{N}$ and $M=N$ ). These dependencies show that the PNR increases, if $M$ increases. They also demonstrate, that the larger is a window

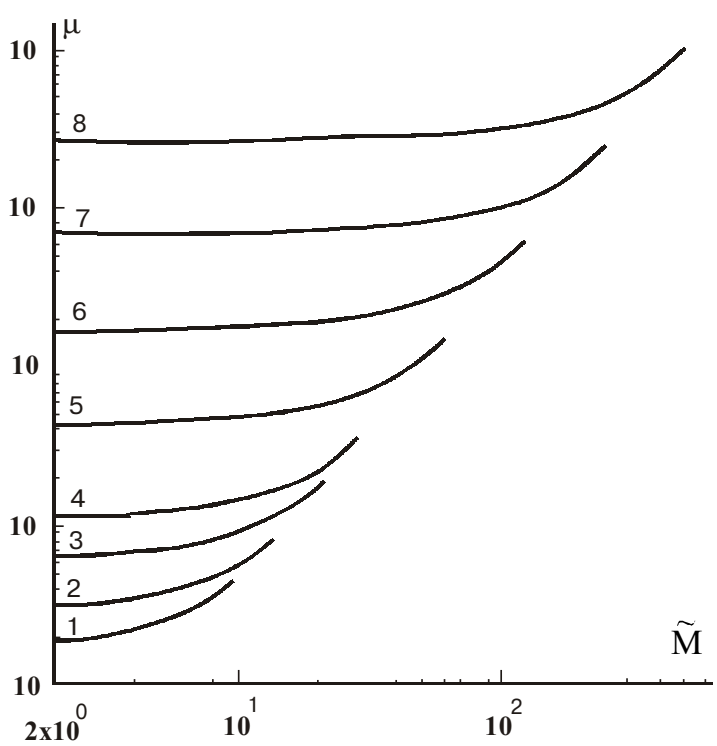

Fig. 2. Dependencies of $\mu$ versus $\tilde{M}$ for $M=12(1) ; \quad M=16$ (2); $\quad M=24$ (3); $\quad M=32$ (4); $M=64$ (5); $M=128$ (6); $M=256(7) ; \quad M=512$ (8).

$(2 \tilde{M}+1) \times(2 \tilde{N}+1)$, the more precise is the estimation of the PNR. On the other hand, the correlation peak intensity sharply decreases with increase of masks dimension, if rotation angles of one mask relatively to another are equal [5]. If transformed phase masks are used in a JTC as optical marks, the dependencies PNR versus dimension of a window $(2 \tilde{M}+1) \times(2 \tilde{N}+1)$ remain approximately the same (see Fig. 2). However, in this case the PNR can essentially decrease because of mutual overlap of an interference noise producing as a result of a crosscorrelation between a transformed mask fragments and a reference mask.

\section{Optically addressed SLM JTC}

To study the performance of transformed PMs as optical marks in an optical JTC containing an OASLM in a frequency domain, we have developed the OASLM JTC experimental setup. In this setup, we used the OASLM based on the chalcogenide glass photoconductor - nematic liquid crystal (CGP-NLC) structure [8]. The reference and transformed PMs were entered to the JTC input plane. The distance between two masks was $2 y_{0}=8 \mathrm{~mm}$. The reference $\mathrm{PM}$ consists of $256 \times 256$ square pixels. The pixel size is $20 \times 20 \mu \mathrm{m}$. The transformed mask consists of several fragments of the reference mask shifted relatively to the initial position by given distance and given direction.

\subsection{Optically adressed SLM}

The OASLM based on the chalcogenide glass photoconductor - nematic liquid crystal (CGP-NLC) structure has high sensitivity and good resolution. High sensitivity (up to $0.01 \mu \mathrm{W} / \mathrm{cm}^{2}$ ) makes it possible to input images in coherent optical processor directly, without an intermedi- 


\section{L.I. Muravsky et al.: Performance of optical and hybrid joint transform...}

ate TV channel. These type of the SLMs have quite good optical quality and low level of spatial noises owing to alignment technique for nematics was recently perfected very well. Response speed of the NLC-SLM is defined by decay time of the NLC that is typically 40-100 ms at LC layer thickness 5-10 $\mu \mathrm{m}$ and time constant of redistribution of the charges in the photoconductor - liquid crystal structure: $\tau \approx R_{P C} C_{L C}$, where $R_{P C}$ is the resistance of the photoconductor layer, $C_{L C}$ is the capacity of the $\mathrm{LC}$ layer. This time constant under typical conditions for the chalcogenide glass semiconductor - nematic liquid crystal structure is about $100 \mathrm{~ms}$. So, usually response speed of the NLC-SLM does not exceed 10-30 Hz.

The SLM based on the CGP-NLC structure consists of number of thin films sandwiched between two glass substrates: a photoconductor, a liquid crystal layer and transparent ITO electrodes. Voltage applied to the ITO electrodes is divided between the photoconductor and the liquid crystal layers according to writing light intensity. Thin films $(1 \mu \mathrm{m})$ of semiconductor $\mathrm{As}_{20} \mathrm{Se}_{80}$ (sensitive in the wide spectral range from 400 to $650 \mathrm{~nm}$ ) were used as a photoconductor. Twisted nematic effect was used for light modulation. Used LC-materials (ZKS-1630B, NIOPIK, Moscow) has the following parameters: $\Delta n=$ $0.13, \Delta \varepsilon=+4.4, \Delta t=-20-+90{ }^{\circ} \mathrm{C}, d=3 \mu \mathrm{m}$. The main characteristics of the transmission mode SLM have been measured in the holographic coherent optical test bench. An interference pattern was written to the SLM and was read by semiconductor laser beam simultaneously. We used a He-Ne laser $(\lambda=633 \mathrm{~nm})$ for writing and a semiconductor laser $(\lambda=814 \mathrm{~nm})$ for reading. We studied the behavior of diffraction efficiency in the first diffraction order as a function of operating conditions. The diffraction efficiency was determined as a ratio of the light intensity in the first order to the reference beam intensity.

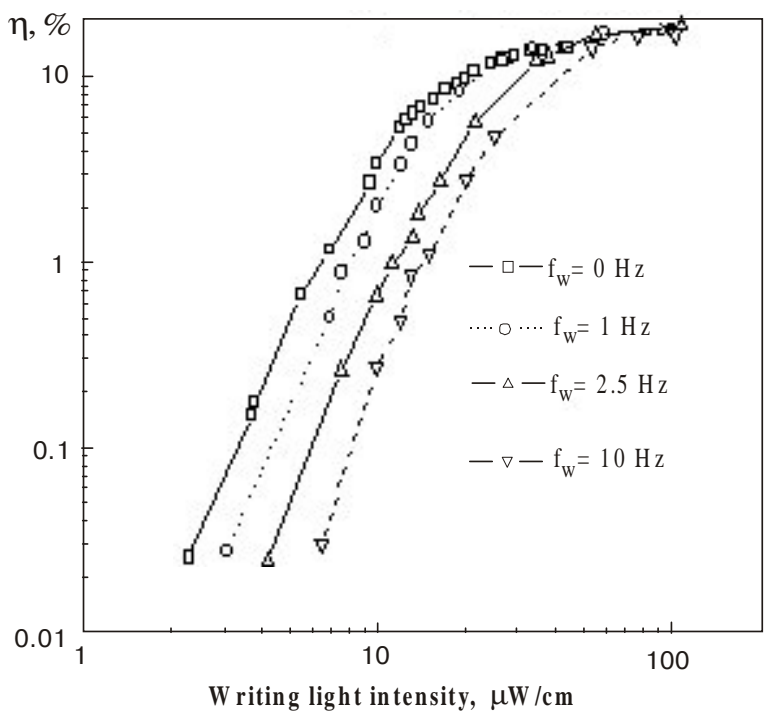

Fig. 3. Diffraction efficiency as a function of writing light intensity at different modulation frequency of writing light (DC voltage, $\mathrm{n}=28 \mathrm{lp} / \mathrm{mm})$.
The special feature of the SLM operating under DC voltage and slowly changed writing light intensity is the high sensitivity to writing light. It is explained by absence of any capacitive current in the PC-LC structure under these conditions. In this case photocurrent is used with maximum efficiency and causes maximum voltage across the LC layer that is defined by conductivity of PC and LC layers. The dependence of diffraction efficiency $\eta$ from writing light intensity under DC voltage $(U=22$ V) and constant illumination is shown in Fig. 3. The sensitivity of the SLM at $\eta=0.1 \%$ is $E=3.3 \mu \mathrm{W} / \mathrm{cm}^{2}$. The sensitivity at $\eta=10 \%$ is $E=11 \mu \mathrm{W} / \mathrm{cm}^{2}$. The maximum diffraction efficiency $\eta=14.7 \%$ is achieved at $E=43$ $\mu \mathrm{W} / \mathrm{cm}^{2}$. The SLM under these conditions has large dynamic range of both writing light intensity and diffraction efficiency that is extremely important when the device is used in optical correlators. The optimal power supply voltage for realization of maximum dynamic range is defined by maximum input light decreasing of intensity. Increasing of writing light intensity from 42 to $430 \mathrm{~mW} / \mathrm{cm}^{2}$ causes the decrease of optimal voltage from 30 to $7.5 \mathrm{~V}$. This decrease of the operating DC voltage results in improvement of dynamic range and decreasing threshold sensitivity of the SLM.

The response speed and real-time recording possibility of the SLM is characterized by dependence of modu-

lation depth of diffraction efficiency $m_{\eta}=\frac{\eta_{\max }-\eta_{\min }}{\eta_{\max }+\eta_{\min }}$ as a function of writing pulse frequency $f_{w}$, where $\eta_{\max }$ is the maximum diffraction efficiency (when writing light is turned on), $\eta_{\text {min }}$ is the minimum diffraction efficiency (when writing light is turned off or dark period). This parameter characterizes decay of recorded grating after turning out writing radiation. Simultaneously with increasing maximum diffraction efficiency, the SLM un-

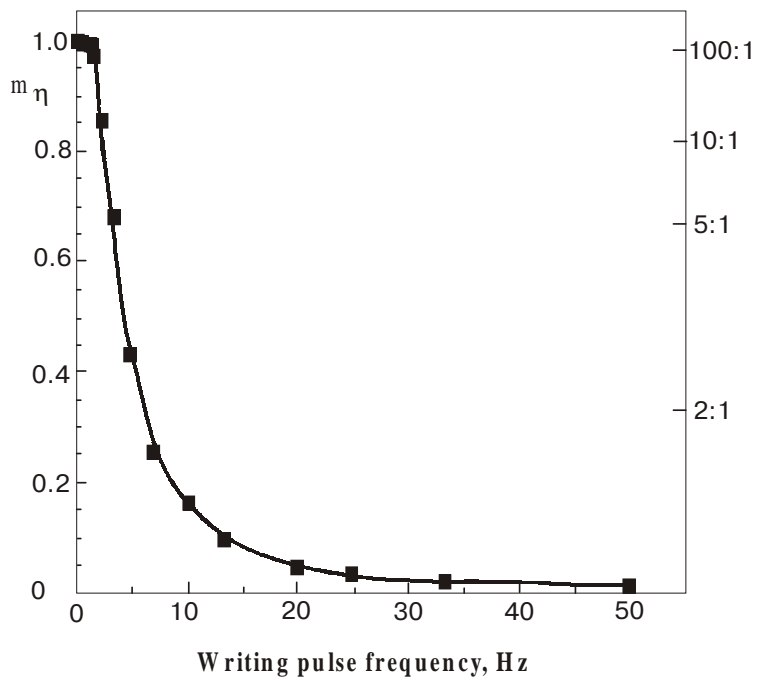

Fig. 4. Modulation depth of diffraction efficiency as a function of writing pulse frequency (DC voltage). The ratio $h_{\max } / h_{\min }$ is shown on the right axis. 


\section{L.I. Muravsky et al.: Performance of optical and hybrid joint transform...}

der modulated writing light displays reduced modulation depth of diffraction efficiency (see Fig. 4). The modulation depth is decreased up to 0.16 at $f_{w}=10 \mathrm{~Hz}$. This means that potential relief on the LC layer does not relax completely during time period between writing light pulses. This process results in a decreasing modulation depth of diffraction efficiency.

\subsection{OASLM JTC experimental setup}

The OASLM JTC experimental setup is shown in Fig. 5. To simplify the scheme of this setup and to provide hereafter creation of the compact security device based on it basis, we used only one laser diode $\operatorname{LD}(\lambda=635 \mathrm{~nm})$ that formed both writing and reading beams. In this setup, the reference PM was built in a special bracket, in which the regulation of a mask position is foreseen. Energizing on the OASLM and recording correlation peaks at the correlator output were provided simultaneously with input of a transformed PM (TPM) in an optical writing beam through the device $S 1$. The writing beam was formed by the LD and the collimator lens (CL). The TPM and reference $\mathrm{PM}$ were illuminated by the writing beam, and a joint Fourier spectrum is produced by a Fourier lens FL1 in a frequency domain of the JTC setup. The OASLM was recorded the produced interference pattern in the JTC frequency domain. The output correlation signal was formed as a result of reading out the recorded interference pattern and its Fourier transform by a Fourier lens FL2. The bright and sharp correlation peaks were formed in the \pm 1 -st diffraction order of the JTC. Thus, the quantity of peaks was equal to quantity of separate fragments in the transformed mask. Each peak was produced as a result of the autocorrelation between the corresponding fragment and the same fragment arranged within the reference PM. The +1 -st diffraction order was recorded by a CCD-camera and entered to a PC using a frame grabber $(\mathrm{FG})$.
Different transformed PMs were studied in this setup. The scheme of the three-fragment transformed mask fabrication procedure that also used in the OASLM JTC, is shown in Fig. 6.

\subsection{Peak-to-noise ratio and relative intensity}

To investigate the performance of this setup and its possibility to identify the optical marks containing the transformed PMs, we studied the PNR as a function of an effective focal length $f_{e}$ of the JTC setup first stage. With this purpose we used two modifications of the JTC first stage, namely: 1) a Fourier lens FL1 before input images, 2) a Fourier lens behind input images. Application of these schemes allowed rather easily to change the effective focal length. In these schemes we have used a divergent lens, due to which we reduced the sizes of the JTC first stage. We calculated the PNR by using the next metrics [4-6]:

$\operatorname{PNR}=\frac{I_{k}-\overline{u_{i, j}}}{\left[\operatorname{var}\left(u_{i, j}\right)\right]^{1 / 2}}$,

where $I_{k}$ is the $k$-th peak intensity, $u_{i, j}$ is the intensity of the surrounded response outside the $50 \%$ peak intensity, $k$ is a number of a peak or number of a transformed PM fragment. We measured the PNR for $21 \times 21$-pixel window centered relatively to each of peaks. The high resolution of the used OASLM allowed to make the PNR measurements in a broad band of effective focal lengths down to $f_{e}=200 \mathrm{~mm}$. The results of the PNR versus $f_{e}$ measurements, when the $256 \times 256$ pixel reference $P M$ and transformed PM (represented in Fig. 6) were used, are listed in Table 1 for the mentioned above two modifications of the JTC first stage. In this Table, the relative intensities $I_{0, k}=I_{k} / I_{d c}$ of correlation peaks, as attitudes of a $k$-th peak intensity $I_{k}$ to a dc intensity $I_{d c}$, also are represented. The relative intensity can be esti-

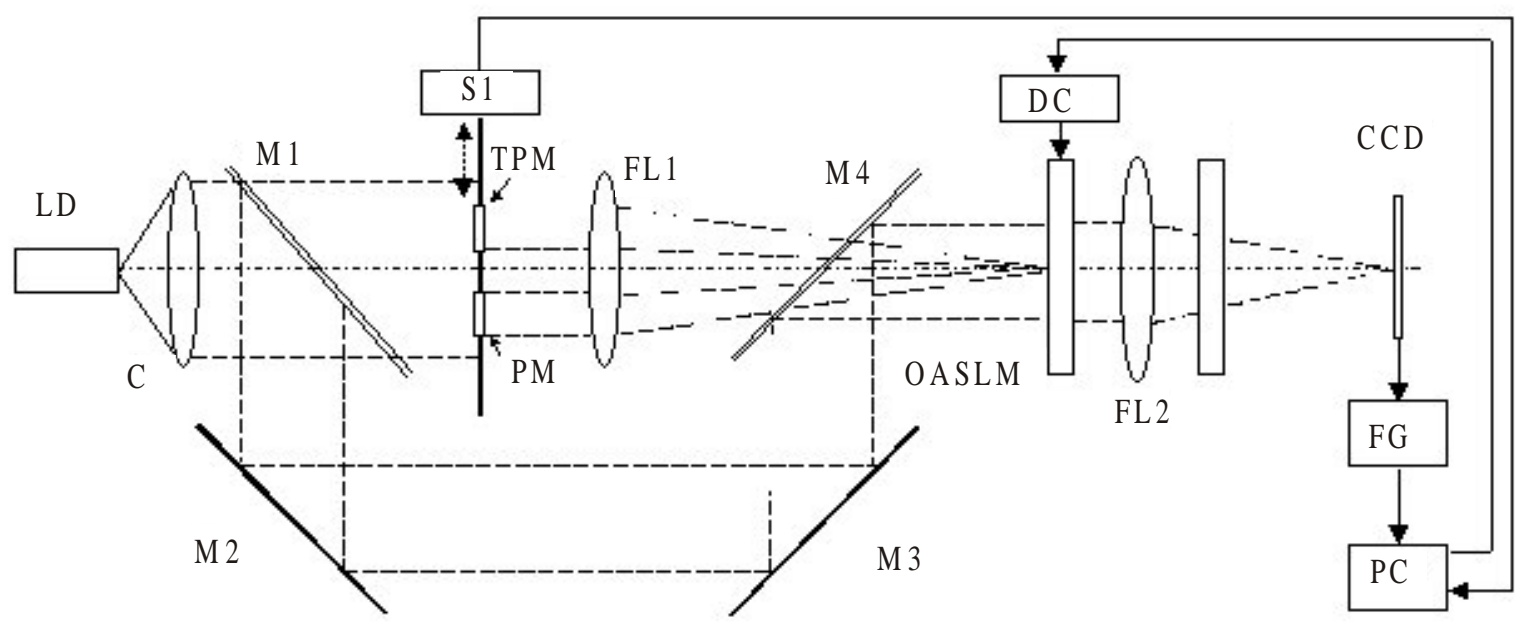

Fig. 5. The OASLM JTC experimental setup: LD - laser diode; C - collimator lens; M1, M4 - beam splitters; M2, M3 - mirrors; PM - phase mask; TPM - transformed phase mask; S1 - switch; FL1, FL2 - Fourier lenses; OASLM - optically addressed SLM based on the CGP-NLC structure; DC - DC voltage; P1 - polarizer; CCD - CCD-camera; FG - frame grabber. 


\section{L.I. Muravsky et al.: Performance of optical and hybrid joint transform...}

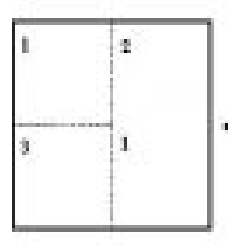

a)

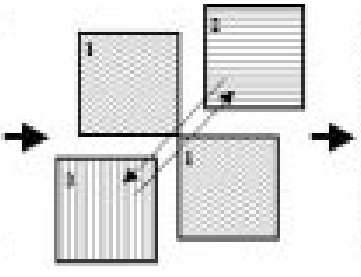

b)

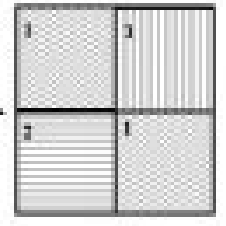

Fig. 6. Scheme of the three-fragment transformed mask fabrication procedure: the reference phase mask (a); separation of the reference PM on fragments (b); the transformed PM (optical mark) (c)

mated as one of possible characteristics of a correlator efficiency. This characteristic is not so obvious, as for example, the Horner efficiency [9], but it is quite reasonable, if an optical and a hybrid optical-digital correlators are compared. Really, the estimation of a hybrid correlator efficiency through the metrics suitable for an estimation of optical correlators is inconvenient, because the intensity of a correlation peak produced in a hybrid correlator digital part and the intensity of a reading beam in an optical correlator are measured in different metrics. On the other hand, the intensity of a DC can be exactly measured both in optical and digital correlator. Moreover, the relative intensity can be easily connected to known estimations of correlator efficiency.

The data represented in Table 1 demonstrate that the downward tendency of PNR is watched with reduction of $f_{e}$. Such tendency is quite natural, as the OASLM resolution is monotonously decreased, if spatial frequency is increased. More difficult is to explain lets for the PNR (for example, in the string \#3, if $f_{e}=430 \mathrm{~mm}$ and in the string \#8 for 3-d peak, if $f_{e}=200 \mathrm{~mm}$ ). We suppose that such lets are connected first of all to phase distortions of correlation signals called rather by poor quality of manufacturing of phase masks and availability of local defects in the OASLM. The correlation response as outcome of a cross-correlation between the three-fragment transformed and reference masks in the output plane $\left(x^{\prime}, y^{\prime}\right)$ of the OASLM JTC, if $f_{e}=400 \mathrm{~mm}$, is adduced in Fig. 7 .

\section{Optoelectronic device}

The optoelectronic device for identification of credit cards is intended for security verification of credit cards and other similar products. This device is built up on the basis of a HOD JTC architecture. It consists of an optical Fourier processor (OFP), a CCD-camera, a frame grabber and a $\mathrm{PC}$ with developed software for realization of the 512×512-pixel Fast Fourier transform (FFT). A trans-

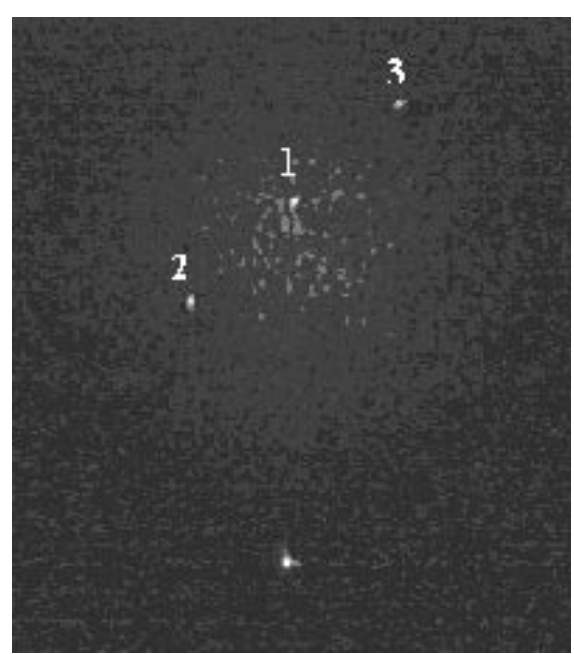

Fig. 7. Correlation response as outcome of a cross-correlation between the three-fragment transformed and reference masks in the output plane $\left(x^{\prime}, y^{\prime}\right)$ of the OASLM JTC for $f_{e}=400 \mathrm{~mm}$ (dc is truncated).

Table 1. Results of the PNR and $I_{0}$ versus $f_{e}$ measurements for the OASLM experimental setup, if the three-fragment transformed PM (see Fig. 6) is used

\begin{tabular}{|c|c|c|c|c|c|c|c|c|c|c|c|c|c|}
\hline \multirow{2}{*}{\multicolumn{2}{|c|}{ Modification }} & \multicolumn{6}{|c|}{ Scheme 1 (before) } & \multicolumn{6}{|c|}{ Scheme 2 (behind) } \\
\hline & & \multicolumn{3}{|c|}{\begin{tabular}{l|l} 
PNR & \\
\end{tabular}} & \multicolumn{3}{|c|}{$I_{0}$} & \multicolumn{3}{|c|}{ PNR } & \multicolumn{3}{|c|}{\begin{tabular}{|r|}
$I_{0}$ \\
\end{tabular}} \\
\hline \multicolumn{2}{|c|}{ \# $\quad$ Focal length $(\mathrm{mm})$} & Peak & \multicolumn{2}{|c|}{ Peak 2 Peak 3} & \multicolumn{3}{|c|}{$\begin{array}{l}\text { Peak } 1 \text { Peak } 2 \text { Peak } 3 \\
\left(\times 10^{-4}\right)\left(\times 10^{-4}\right)\left(\times 10^{-4}\right)\end{array}$} & \multirow[t]{2}{*}{ Peak 1} & \multicolumn{2}{|c|}{ Peak 2 Peak 3} & \multicolumn{3}{|c|}{$\begin{array}{l}\text { Peak } 1 \text { Peak } 2 \text { Peak } 3 \\
\left(\times 10^{-4}\right)\left(\times 10^{-4}\right)\left(\times 10^{-4}\right)\end{array}$} \\
\hline 1 & 530 & 10.7 & 11.2 & 14.4 & 12 & 8.1 & 6.3 & & & & & & \\
\hline 2 & 450 & 8.8 & 8.8 & 10.2 & 12 & 8.3 & 6.2 & & & & & & \\
\hline 3 & 430 & 10.1 & 10.8 & 18.0 & 12 & 8.4 & 6.2 & & & & & & \\
\hline 4 & 400 & 10.2 & 8.3 & 11.2 & 12 & 8.5 & 6.2 & 11.3 & 10.1 & 14.8 & 13 & 8.7 & 7.2 \\
\hline 5 & 350 & & & & & & & 9.5 & 10.6 & 14.7 & 13 & 9.1 & 6.7 \\
\hline 6 & 300 & 12.4 & 12.0 & 10.1 & 12 & 9.6 & 7.1 & 8.0 & 10.5 & 12.6 & 12 & 9.8 & 7.5 \\
\hline 7 & 250 & & & & & & & 8.0 & 9.3 & 10.7 & 12 & 9.2 & 3.3 \\
\hline 8 & 200 & 9.1 & 9.1 & 15.9 & 6.1 & 5.3 & 5.1 & 10.1 & 11.8 & 4.5 & 11 & 9.2 & 1.5 \\
\hline
\end{tabular}




\section{L.I. Muravsky et al.: Performance of optical and hybrid joint transform...}

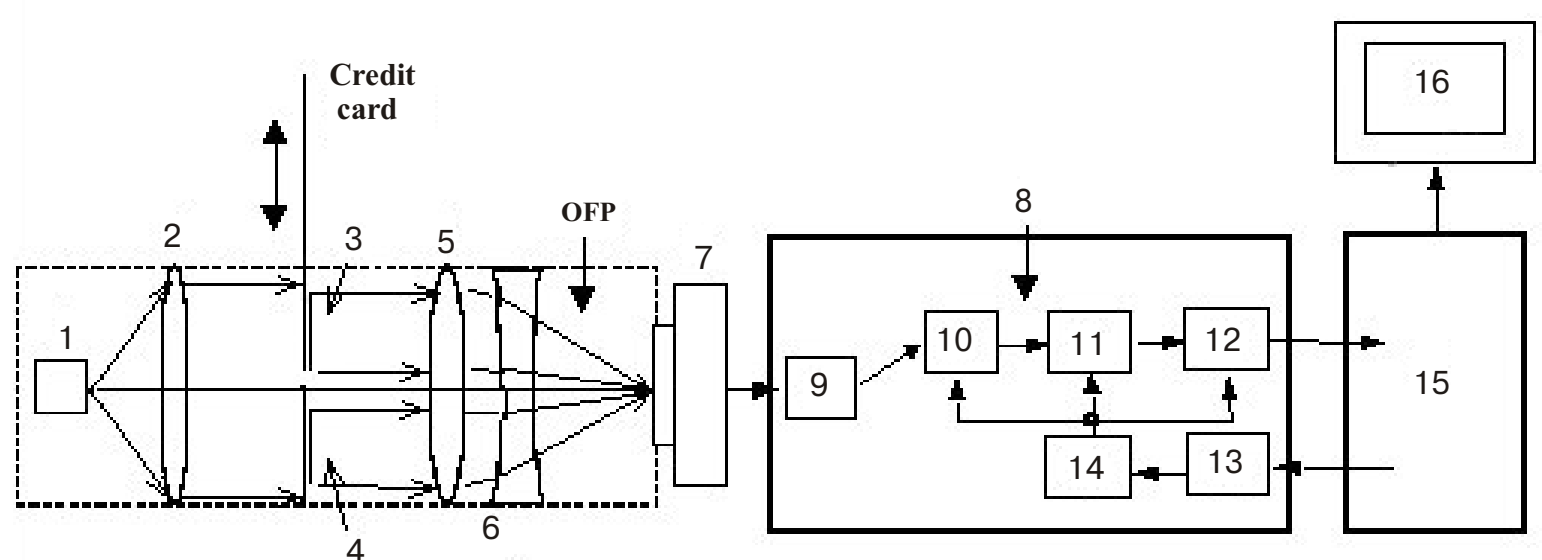

Fig. 8. Scheme of the optoelectronic device: OFP - optical Fourier processor, 1 - laser diode (l=635 nm), 2 - collimator lens, 3 transformed PM (optical mark), 4 - reference PM, 5 - Fourier lens, 6 - divergent lens, 7 - CCD-camera, 8 - frame-grabber, 9 - video amplifier, 10 - ADC, 11 - RAM, 12 - data bus, 13 - address bus, 14 - signal synchronizer, 15 - PC, 16 - display.

formed PM is used in this device as an optical mark bonded to a credit card to be identified.

The scheme of the device is shown in Fig. 8. The laser beam is generated by a laser diode $1(\lambda=635 \mathrm{~nm})$ and is collimated by a collimator 2 . The collimated laser beam illuminats the transformed PM 3 and the reference PM 4 placed in the input plane of the OFP. The reference mask is strongly attached to the special holder in the OFP input plane. The transformed mask is entered into the OFP input plane as the optical mark bonded to the credit card. A joint Fourier transform spectrum is formed by the Fourier lens 5 and is produced on the OFP output as a result of a joint Fourier transform of both masks. The CCD-camera 7 records the joint power spectrum. The interference pattern is captured by the frame grabber 8 and is converted into a digital massif with a $512 \times 512$ pixels dimension. The modified FFT algorithm is used to perform the inverse Fourier transform in the PC 15. If the optical mark is authentic, the several narrow and bright correlation peaks are produced in the first diffraction order of the JTC output, and quantity of peaks is equal to quantity of fragments of the transformed PM. Calculated distances between peaks form an identification feature vector $(\mathrm{FV})$ which is compared with a reference FV [6]. If the distance $D$ between the identification and reference FVs is less than the given distance $D_{0}$, the credit card is considered authentic. Then the identification signal is emerged on the display 16. A divergent lens 6 is used to change the effective focal length $f_{e}$ of the OFP.

To define minimum permissible focal length $f_{N, k}$ of the Fourier transform lens in the OFP, at which the recovery of the $k$-th correlation peak without a loss of information still agrees with the sampling theorem [10], we have used the following equation:

$$
f_{N, k}=\frac{\left(2 p_{y}+p_{x} \operatorname{tg} \beta_{k}\right) \cos \beta_{k}\left|\left(\vec{l}_{k}\right)\right|}{\lambda}
$$

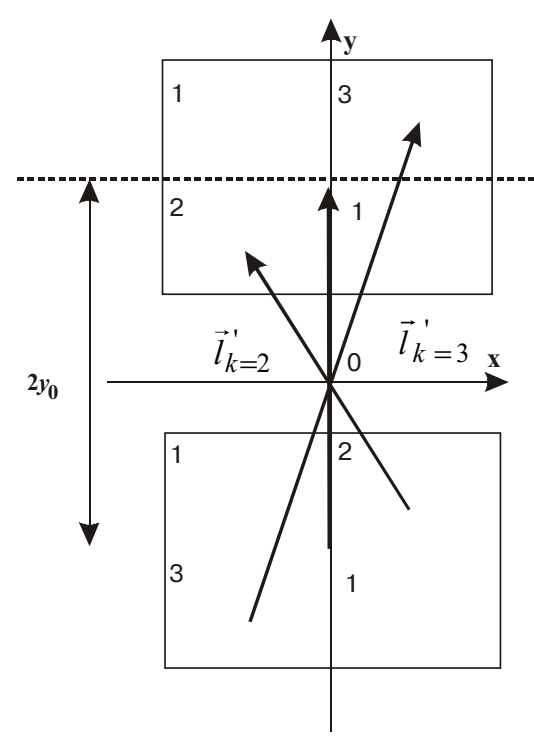

Fig. 9. Arrangement of the vector in a JTC input plane $(x, y)$.

where $p_{x}$ and $p_{y}$ are the pitches between pixels in the CCD matrix in horizontal and vertical directions accordingly; $\vec{l}_{k}$ is the vector arranged in an input plane of a correlator, this vector connects center of a $k$-th fragment in a reference mask to center of the same $k$-th fragment in the transformed mask (see Fig. 9); $\beta_{k}$ is the angle between vector $\vec{l}_{k}$ and axis $\vec{y}$ of the JTC input plane $(x, y)$. Note, that $\beta_{k}$ is equal to the angle between vector $\vec{l}_{k}^{\prime}$ from a point about the origin of the JTC output plane $\left(x^{\prime}, y^{\prime}\right)$ up to the point of the $k$-th correlation peak maximum and axis $\vec{y}^{\prime}$ of the output plane (see Fig. 10). If the scales of direct and inverse Fourier transforms in the JTC are identical, then $\vec{l}_{k}=\vec{l}_{k}^{\prime}$.

Different transformed PMs were tested in the optoelectronic device. One of these masks is represented 


\section{L.I. Muravsky et al.: Performance of optical and hybrid joint transform...}

Table 2. Calculation of the minimum focal length $f_{N, k}$ of the Fourier transform lens in the OFP for the three-fragment transformed PM.

\begin{tabular}{lllll|l}
\hline \hline$k$ & $x_{k}, \mathrm{~mm}$ & $y_{k}-2 y_{0}, \mathrm{~mm}$ & $\beta_{k}, \mathrm{deg}$ & $\vec{l}_{k} \mid, \mathrm{mm}$ & $f_{N, k}, \mathrm{~mm}$ \\
\hline 1 & 0 & 0 & 0 & 8.000 & 202.2 \\
\hline 2 & 2.560 & -2.560 & 25.20 & 6.012 & 169.9 \\
\hline 3 & -2.560 & 2.560 & 13.63 & 10.867 & 299.3 \\
\hline \hline
\end{tabular}

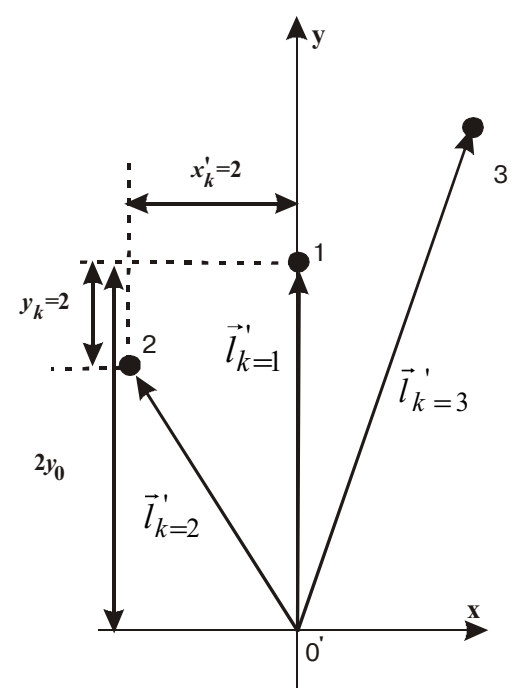

Fig. 10. Arrangement of the vectors in a JTC output plane $\left(x^{\prime}, y^{\prime}\right)$.

Table 3. Results of the PNR and relative intensity $I_{0}$ measurements for the optoelectronic device based on a HOD JTC architecture, if the three-fragment transformed PM (see Fig. 6) is used.

\begin{tabular}{|c|c|c|c|c|c|c|c|c|}
\hline & \multirow[t]{2}{*}{$\#$} & \multirow[t]{2}{*}{ Quadrant } & \multicolumn{3}{|c|}{ PNR } & \multicolumn{3}{|c|}{$I_{0}$} \\
\hline & & & Peak 1 & Peak 2 & Peak 3 & Peak 1 & Peak 2 & $\overline{\text { Peak } 3}$ \\
\hline \multirow{4}{*}{$\begin{array}{l}\text { Direct recording } \\
\text { of an interference } \\
\text { pattern }\end{array}$} & 1 & $1-4$ & 27.7 & 19.5 & 23.8 & 0.25 & 0.09 & 0.15 \\
\hline & 2 & 3,4 & 24.9 & 14.1 & 44.8 & 0.22 & 0.07 & 0.25 \\
\hline & 3 & 1,2 & 34.1 & 16.1 & 41.8 & 0.26 & 0.06 & 0.18 \\
\hline & 4 & 1 & 34.4 & 16.1 & 31.1 & 0.52 & 0.12 & 0.31 \\
\hline \multirow{3}{*}{$\begin{array}{l}\text { Fourier plane } \\
\text { image subtraction } \\
\text { technique }\end{array}$} & 5 & 3,4 & 8.1 & 16.4 & 14.3 & 0.13 & 0.09 & 0.16 \\
\hline & 6 & 1,2 & 7.9 & 13.7 & 18.3 & 0.06 & 0.07 & 0.18 \\
\hline & 7 & 1 & 33.6 & 7.1 & 7.1 & 0.25 & 0.07 & 0.07 \\
\hline \multirow{2}{*}{$\begin{array}{l}\text { Power spectrum } \\
\text { subtraction } \\
\text { technique }\end{array}$} & 8 & 1,2 & 25.5 & 23.1 & 23.1 & 0.19 & 0.31 & 0.31 \\
\hline & 9 & 1 & 15.1 & 22.4 & 14.1 & 0.31 & 0.25 & 0.23 \\
\hline
\end{tabular}

in Fig. 6. The calculated quantities of minimum allowable focal length $f_{N, k}$ for all three correlation peaks produced at the optoelectronic device output as a result of a cross-correlation between this mask and the reference PM are represented in Table 2. In this Table, $x_{k}$ and $y_{k}$ are projections of the vector $\vec{l}_{k}$ (see Fig. 9). This Table shows that minimum focal length of the Fourier transform lens for this mask corresponds to $f_{N, k=3}=299.3 \mathrm{~mm}$.

To compare performances of the OASLM JTC and optoelectronic device based on a HOD JTC, we used the same masks in the optoelectronic device. We calculated the PNR at the output of the optoelectronic device by using the same Eq. (11), as for calculation of the PNR in SQO, 5(2), 2002

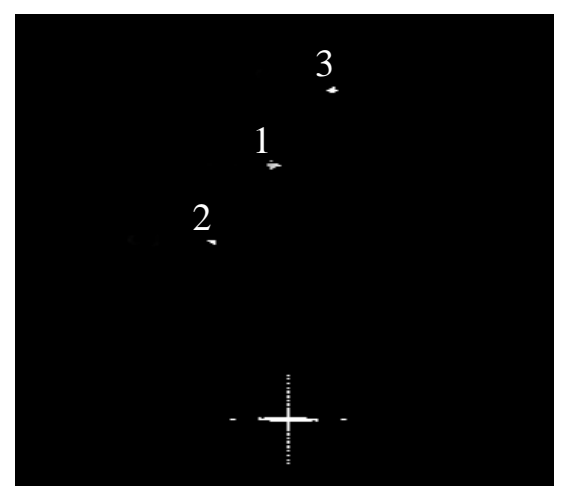

Fig. 11. Correlation response as outcome of a cross-correlation.

the OASLM JTC. The results of the PNR calculations for $f_{e}=400 \mathrm{~mm}$ are represented in Table 3 . This Table also represents the relative intensities $I_{0, k}$ of the correlation peaks.

The results of the PNR and relative intensity $I_{0}$ measurements are represented in Table 3 for the next conditions of the interference pattern recording: string \#1 - the CCD-camera records the center of the interference pattern (1 - 4-th quadrants of a frequency domain $\left(v_{x}, v_{y}\right)$ ); string \#2 - the bottom of the interference pattern (3-d and 4-th quadrants); string \#3 - the top of the interference pattern (1-st and 2-nd quadrants), string \#4 - the upper right hand part of an interference pattern (1-st quadrant). So, the PNR and $I_{0}$ are different, if the different parts of 


\section{L.I. Muravsky et al.: Performance of optical and hybrid joint transform...}

the same interference pattern are recorded by the CCDcamera. Such different values of the PNR and $I_{0}$, first of all, should be explained by a high level of an optical noise in the optoelectronic device. The correlation response as outcome of a cross-correlation between the three-fragment transformed and reference masks in the output plane $\left(x^{\prime}, y^{\prime}\right)$ of the optoelectronic device $\left(f_{e}=400 \mathrm{~mm}\right)$ is adduced in Fig. 11. This responce was obtained by recording of the third and fourth quadrants of a frequency domain $\left(v_{x}, v_{y}\right)$.

We also investigated the effectiveness of a Fourier plane image subtraction technique [11] in the optoelectronic device (see to graph «Fourier plane image subtraction technique» in the Table 3). The made attempts of its application were not crowned with success, to what the outcomes induced in strings \#5-7 as Table 3 testifies. The unexpected and interesting outcomes were obtained in that case, when we subtracted the power spectrum $S^{2}\left(v_{x}, v_{y}\right)$ of the transformed mask complex amplitude transmittance $s(x, y)$ from the power spectrum $R^{2}\left(v_{x}, v_{y}\right)$ of the reference mask's transmittance $r(x, y)$ (see to graph «Power spectrum subtraction technique»). Now we spend more in-depth studies of this effect.

\section{Conclusions}

The comparative analysis of Table 1 and Table 3 shows that the PNR and relative intensity $I_{0}$ in the optoelectronic device are much above, than in the OASLM JTC. The low PNR and $I_{0}$ in the OASLM JTC are explained to that we have used the recording and reading beam from the same laser diode. The preliminary researches have shown that usage in a JTC of a reading beam on a wavelength $\lambda=814 \mathrm{~nm}$ results in approximately three-fold increase of a PNR. Let's also note, that the rather low-level values of the PNR for both JTC types in comparison with theoretically calculated PNR values (see Fig. 2) are explained by poor quality of a substrate, on which the phase masks were produced, as well as by a high level of optical noise in both correlators.

Thus, it is possible to assert that security devices for identification of phase masks can be created on the basis of architectures of both hybrid and optical correlators. However, it is necessary to allow for features of creation of such devices. So, for example, if we design the optical security devices on the basis architectures of an optical correlator, the main attention should be given to research of operating characteristics of the SLM and selection of its optimum regimes in the device. Thus, the main design problem for creation of such devices will be reduction their overall dimensions. If we design the hybrid security devices, the main attention should be paid on mining of the interface and operating FFT-processor. The overall dimensions of such devices will be determined in the main scale of the optical scheme. Therefore, basic requirement to the optical scheme in OFP consists in achievement of the largest possible effective focal distance at minimum allowable overall OFP dimensions.

\section{Acknowledgments}

The authors are grateful to M.V. Shovgenyuk, T.V. Fityo and P.A. Hlushak for fruitful discussions and technical support.

\section{References}

1. B. Javidi and J.L. Horner, Optical pattern recognition for validation and security verification // Opt. Eng. 33(6), pp.1752-1756 (1994)

2. B. Javidi, G. Chang, and J. Li, Experimental demonstration of the random phase encoding technique for image encryption and security verification // Opt. Eng. 35(9), pp.2506-2512 (1996).

3. L.I. Muravsky, V.M. Fitio, M.V. Shovgenyuk, and P.A. Hlushak, Separation of random phase mask in optical correlator for security verification // SPIE Proc. 3466, pp.267277 (1998).

4. L.I. Muravsky, T.I. Voronyak, V.M. Fitio, and M.V Shovgenyuk, Transformed phase mask and photoanisotropic material in optical correlators applied for security verification // Opt. Eng. 38(1), pp.25-32 (1999).

5. L.I. Muravsky, Binary phase image processing in optical and optical-digital correlation systems, Serpen', Ternopil', 1999 (in Ukrainian).

6. L.I. Muravsky, Ya. P. Kulynych, T. I. Voronyak, V. M. Fityo, and S. A. Kostyukevych, Transformed phase mask in a hybrid joint transform correlator for security verification // SPIE Proc. 3804, pp.233-240 (1999).

7. V.M. Fitio, L.I. Muravsky, and A.I. Stefansky, Using of random phase masks for image recognition in optical correlators // SPIE Proc. 2647, pp.224-234 (1995).

8. F.L. Vladimirov, A.N. Chaika, N.I. Pletneva, I.E. Morichev, and N. Collings, Spatial light modulators based on chalcogenide glass photoconductor - liquid crystal structure operating in pulse regime // SPIE Proc. 3490, pp.432 - 435 (1998).

9. J.L. Horner, Metrics for assessing pattern-recognition performance // Appl. Opt. 31(2), pp.165-166 (1992).

10. R.N. Bracewell, The Fourier Transform and Its Applications, Mc Graw-Hill Book Company, New York, 1965.

11. M.A. Alam, and M.A. Karim, Multiple target detection using a modified fringe-adjusted joint transform correlator // Opt. Eng. 33(5), pp.1610-1617 (1994). 